\title{
Static Performance Analysis of Water-Lubricated Hydrostatic-Dynamic Hybrid Bearing Regulated by Multi-Head Pump
}

\author{
Jianhua Zhao ${ }^{\text {* }}$ and Dianrong Gao ${ }^{\mathrm{b}}$ \\ Hebei Provincial Key Laboratory of Heavy Machinery Fluid Power Transmission and Control of \\ Yanshan University, Qinhuangdao 066004 \\ azhaojianhua@ysu.edu.cn, bogadr@ysu.edu.cn
}

Keywords: Hydrostatic-Dynamic Hybrid Thrust Bearing; Multi-Head Pump; Opposed Ladder Pocket; Static Bearing Force; Dynamic Bearing Force

\begin{abstract}
Due to the complex structure, the difficult calculation process of the bearing force and many influence factors of static performance of Water-Lubricated Hydrostatic and Dynamic Hybrid Bearing, the static performance index and influence rule are hardly to be explored and mastered by the engineer, and then it has brought great difficulty to the design of the bearing. Therefore, the paper derives the bearing core equations of single fan-shape ladder-pocket support pad and opposed bearing system, and analyzes the influence law of the design parameters on the static performance of Hydrostatic and Dynamic Hybrid Bearing System regulated by Multi-Head Pump. The result shows that the bearing force is composed of static force and dynamic force, and the dynamic force does not affect the static performance. The bearing capacity increases gradually with the increase of pump pressure and the film thickness, and decreases with the increase of shadow pocket thickness, which has nothing to do with the flow rate and rotational speed. The static stiffness increases gradually with the increase of pump pressure, and decreases with the increase of shadow pocket thickness and film thickness, which has nothing to do with flow rate and rotational speed. The power loss increases gradually with the increase of flow rate, pump pressure and rotational speed, and decreases with the increase of shadow pocket thickness and film thickness. The proposed research provides the theoretical reference for the design and analysis of the Hydrostatic and Dynamic Hybrid Bearing system.
\end{abstract}

\section{Introduction}

There are static-pressure sealing belt and dynamic-pressure wedge groove in the Water-Lubricated Hydrostatic-Dynamic Hybrid Bearing. It can integrate with static bearing effect and dynamic bearing effect, and has the advantages of superior bearing capacity and high stiffness [1-2]. Multi-Head Pump supports the lubricating oil by using the method of constant flow, and then ensures that the flow of hydrostatic system cannot be affected by the external load.

Lots of scholars and researchers have done great investigation on hydrostatic bearing and multi-head pump [3]. WANG [4] discussed the impact of the initial oil film on static and dynamic performances of liquid thrust bearing oil-supply method and they have been verified by experiment. LIU[5] and WANG[6] expounded the advantage of hydrostatic guideway quantitative oil-supply, analyzed the static and dynamic property of long pump oil hydrostatic guideway, and it was the development direction of Heavy duty machine tool motion guideway. GUO, LIU [7-8] analyzed the hydrostatic guide ways on heavy duty vertical lathe and CNC cylindrical grinder, and introduced the quantitative hydrostatic guideway design process and the selection of related parameters.

At present, scholars mainly focus their study on the performance analysis of the hydrostatic-dynamic bearing and multi-head pump.

Due to the complex structure, the difficult calculation process of the bearing force and many influence factors of static performance of Water-Lubricated Hydrostatic-Dynamic Hybrid Bearing, the static performance index and influence rule are hardly to be explored and mastered by the engineer, 
and then it has brought great difficulty to the design of the bearing. Up to now, there is no effort toward the research static performance of hydrostatic-dynamic hybrid bearing supported by multi-head pump.

Therefore, the paper derives the bearing core equations of single fan-shape ladder-pocket support pad and opposed bearing system, and analyzes the influence law of the design parameters on the static performance of Hydrostatic and Dynamic Hybrid Bearing System regulated by Multi-Head Pump.

\section{Profile of Multi-head Pump-Hybrid Bearing System}

The hydrostatic-dynamic hybrid bearing supported by multi-head pump is as shown inin Fig. 1 .

There are six axial and radial support pads in the Water Lubricated Hydrostatic Dynamic Hybrid Bearing, as shown in Fig. 2. Support pad is composed of inlet, ladder pocket, water sealing belt, central bearing zone, return flume, return hole and so on.

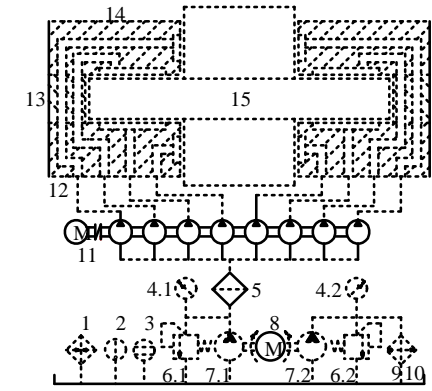

Fig. 1 Sketch of hydrostatic-dynamic hybrid bearing supported by multi-head pump

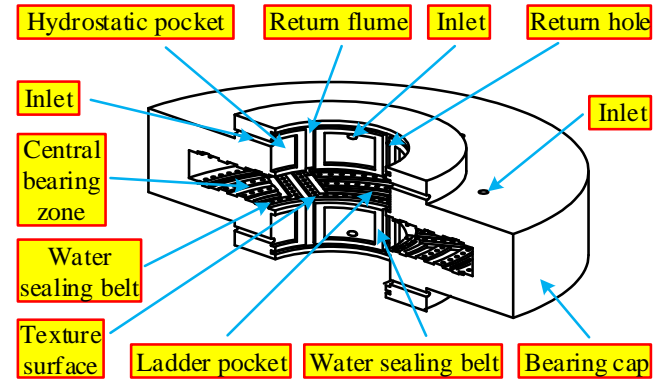

Fig. 2 Half-section sketch of bearing cap

Multi-head pump is composed of cap, main body, plunger, eccentric crankshaft, support bearing, rotating bearing, inlet, and return hole. Flow of lubricant can be generated evenly through radial motion of plunger, and the stiffness of flow can't be affected by the load.

\section{Bearing Force of Hydrostatic-dynamic Support Pad}

Bearing force of single fan-shape ladder-pocket support pad. There are hydrostatic water sealing belt and ladder pocket in the water lubricated bearing, and the bearing can integrate with dynamic pressure effect and hydrostatic pressure effect. According to Reference [9], the bearing force of single fan-shape support pad can be shown as follow:

$$
F=F_{j}+F_{d}
$$

Where, $\mathrm{Fj}$ is hydrostatic bearing force, and $\mathrm{Fd}$ is dynamic bearing force. Hydrostatic bearing force is mainly formed through throttling effect of water sealing belt, and dynamic bearing force is mainly formed through velocity difference and wedge dynamic effect between top and bottom bearing cap. The expressions are shown as follow:

$$
\left\{\begin{array}{l}
F_{j}=p_{j} A_{e, j} \\
F_{d}=p_{d} A_{e, d}
\end{array}\right.
$$

Where, $p_{j}, p_{d}, A_{e, j}, A_{e, d}$ are respectively inlet pressure and equivalent bearing area.

Bearing force of opposed fan-shape ladder-pocket support pad. For the sake of calculation and analysis, the paper mainly analyzed one opposed fan-shape ladder-pocket support pad of water-lubricated bearing.

Bearing force is composed of hydrostatic bearing force and dynamic bearing force. However, the bearing force of hydrostatic-dynamic hybrid bearing regulated by multi-head pump is only affected by hydrostatic bearing force. The bearing force is shown as follows: 


$$
f=\left.2 q_{0} A_{e} \frac{\partial \Omega_{26}}{\partial h}\right|_{h=h_{0}} \Delta h
$$

Where, $\Omega 26,0$ is hydraulic resistance when the film thickness is h0, dimensionless.

\section{Static Performance Index of Opposed Support Pad}

The static performance index of water-lubricated hydrostatic-dynamic hybrid bearing is composed of bearing capacity, static stiffness and power loss.

Bearing Capacity. Bearing capacity F0 characterizes the max external load that the hybrid bearing can bears when film thickness is zero, and its expression is shown as follows:

$$
F_{0}=\left.2 q_{0} A_{e} h_{0} \frac{\partial \Omega_{26}}{\partial h}\right|_{h=h_{0}}
$$

Static Stiffness. Static stiffness j0 characterizes the ability of the film to resist deformation under the external load, and its expression is shown as follows:

$$
j_{0}=-\left.\frac{\partial F}{\partial h}\right|_{h=h_{F}}=\left.2 q_{0} A_{e} \frac{\partial \Omega_{26}}{\partial h}\right|_{h=h_{0}}
$$

Where $F-$ External load, N;

$h_{F}-$ Film thickness under external load, $\mathrm{m}$.

Power Loss. Power loss W (Except overflow loss) characterizes the sum of pump power and friction power of hydrostatic pocket, and its expression is shown as follows:

$$
W=\mu v^{2}\left(\frac{A_{s}}{h}+\frac{A_{t}}{h+h_{p}}\right)+p_{s} q
$$

Where, $\mu-$ Dynamic viscosity of lubricant , Pa.s;

$v$-Equivalent velocity, $\mathrm{m} / \mathrm{s}$;

$A_{s} \_$Area of water sealing belt, $\mathrm{m} 2$;

$A_{t}$ Area of shallow pocket, $\mathrm{m} 2$;

$h$-Film thickness of water sealing belt, $\mathrm{m}$;

$h_{p}$-Depth of shallow pocket, $\mathrm{m}$.

Parameter affecting the static performance can be adjusted in the design process, and the operation is composed of rotating angular velocity, pump pressure, flow, shallow pocket depth and film thickness.

\section{Influence of Parameter on Static Performance Index}

\begin{tabular}{|c|c|c|c|c|c|c|}
\hline Parameter & Pump pressure $p_{s} / \mathrm{MPa}$ & Pocket pressure $p_{0} / \mathrm{MPa}$ & Film thickness $h_{0} / \mu \mathrm{m}$ & \multicolumn{3}{|c|}{ Hydraulic resistance $R /$ dimensionless } \\
\hline Value & 10 & 3 & 30 & \multicolumn{3}{|c|}{$1.5501 \times 10^{-6}$} \\
\hline Parameter & Rotating velocity $\omega(\mathrm{r} / \mathrm{min})$ & Flow $Q /(\mathrm{L} / \mathrm{min})$ & ubricant temperature $t /^{\circ} \mathrm{C}$ & \multicolumn{3}{|c|}{ Dynamic viscosity $\eta / \mathrm{Pa} \cdot \mathrm{s}$} \\
\hline Value & 2700 & 11.0045 & 20 & \multicolumn{3}{|c|}{$1.005 \times 10^{-3}$} \\
\hline \multicolumn{7}{|c|}{$\begin{array}{c}\text { The structure and parameters of fan-shape ladder pocket is shown in Tab.2 and Fig. } 4 . \\
\text { Table } 2 \text { Parameter of Fan-shape Ladder Pocket }\end{array}$} \\
\hline Parameter & Film thickness $h / \mu \mathrm{m}$ & Shallow pocket Depth $h_{p} / \mu \mathrm{m}$ & Size $R_{1} / \mathrm{mm}$ & Size $R_{2} / \mathrm{mm}$ & Size $R_{3} / \mathrm{mm}$ & Size $R_{4} / \mathrm{mm}$ \\
\hline Value & 30 & 15 & 45 & 50 & 95 & 100 \\
\hline Parameter & Angle $\theta /^{\circ}$ & Angle $\theta_{e} /^{\circ}$ & Angle $\theta_{0} /^{\circ}$ & Angle $\theta_{1} /{ }^{\circ}$ & Angle $\theta_{2} /^{\circ}$ & Angle $\theta_{3} /^{\circ}$ \\
\hline Value & 30 & 55 & 10 & 5 & 5 & 40 \\
\hline
\end{tabular}

Parameter of hydrostatic-dynamic hybrid bearing. The parameter of hydrostatic-dynamic hybrid bearing is shown in Tab.1.

Table 1 Parameters of Hydrostatic-dynamic Hybrid Bearing 
Influence of Flow on Static Performance. As the flow increases gradually, the bearing capacity, static stiffness and power loss of single support pocket increase synchronously, but the difference between two opposed support pockets remain the same. Therefore, the bearing capacity and static stiffness of two opposed support pockets are not affect by the flow, and the power loss increases with the flow, as shown in Fig. 5.

As the rotating velocity increases gradually, bearing capacity, static stiffness and power loss do not affect by the rotating velocity, as shown in Fig. 5.

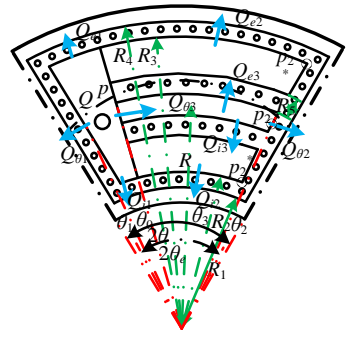

Fig. 4 Sketch of Fan-shape Ladder Pocket

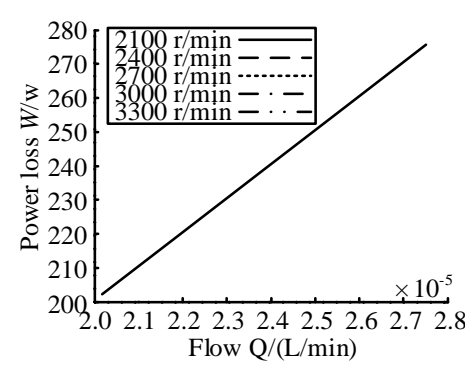

Fig. 5 Relationship between Power Loss and Flow

Influence of pump pressure on static performance. As the pump pressure increases gradually, the bearing capacity and static stiffness of two opposed support pockets increase synchronously, as shown in Fig. 6.

As the pump pressure increases gradually, the overflow and throttle loss become bigger, and then power loss of two opposed support pockets increase synchronously. As the rotating velocity increases gradually, the power loss change a little, and then can be basically ignored as shown in Fig. 7.

Influence of shallow pocket on static performance. As the shallow pocket depth increases, the depth difference between the shallow pocket and deep pocket gradually decreases, and then the dynamic effect that generate when lubricant flow through ladder position decreases, so bearing capacity and static stiffness of two opposed support pockets decrease synchronously, as shown in Fig. 8.

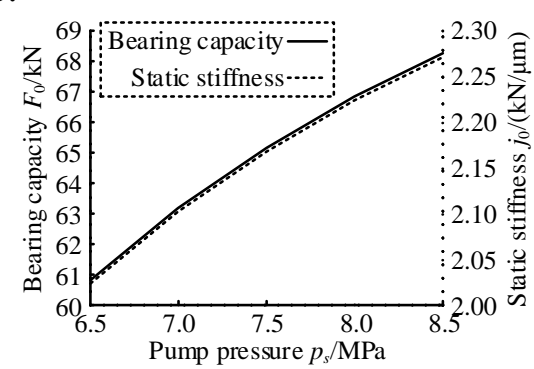

Fig. 6 Relationship between bearing capacity, static stiffness and pump pressure

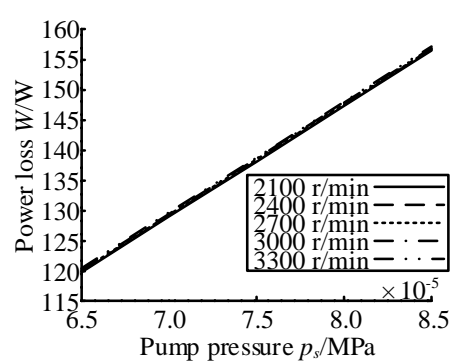

Fig. 7 Relationship between power loss and pump pressure

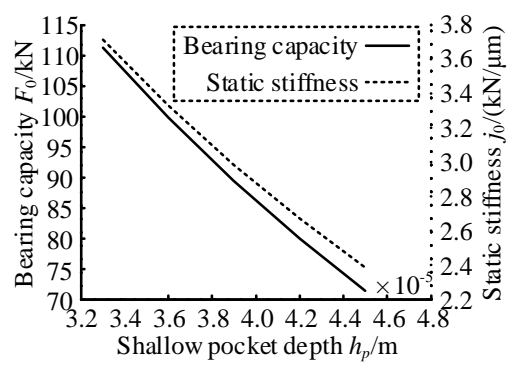

Fig. 8 Relationship between bearing capacity, stiffness and shallow pocket

As the shallow pocket depth increases gradually, the hydraulic resistance become smaller, and then throttle loss that generates when lubricant flows through shallow pocket decreases. However, the range of the change is small, and it can be basically ignored. As the rotating velocity increases gradually, the dynamic bearing effect become bigger, the wedge squeeze effect increases, and then heat budget and power loss of two opposed support pockets increase synchronously as shown in Fig. 9.

Influence of film thickness on static performance. When the anti-thrust ring reaches to the limit position, as film thickness increases gradually, the hydraulic resistance and bearing force of one support pocket will become smaller, and then bearing capacity of two opposed support pockets increase synchronously. As film thickness increases gradually, and static stiffness of two opposed support pockets decrease synchronously as shown in Fig. 10.

As the film thickness increases gradually, the hydraulic resistance of support pocket and energy loss of lubricant become bigger, and then power loss of bearing system increases. As the rotating velocity 
increases gradually, the power loss of two opposed support pockets increase synchronously, but the range can be basically ignored as shown in Fig. 11.

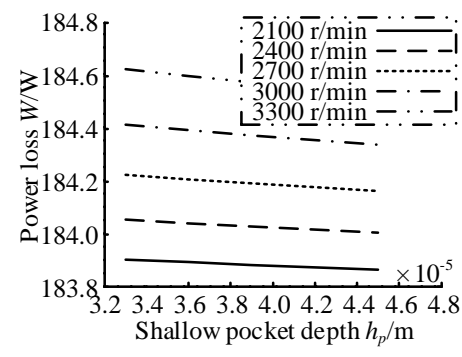

Fig. 9 Relationship between power loss and shallow pocket

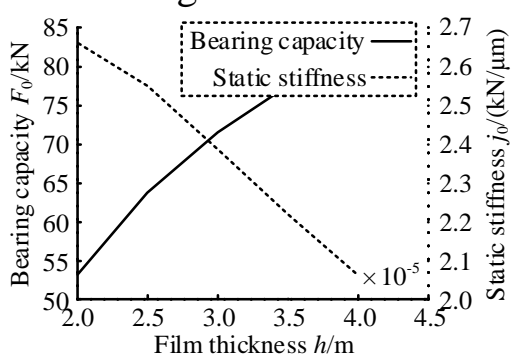

Fig. 10 Relationship between bearing capacity, static stiffness and film thickness

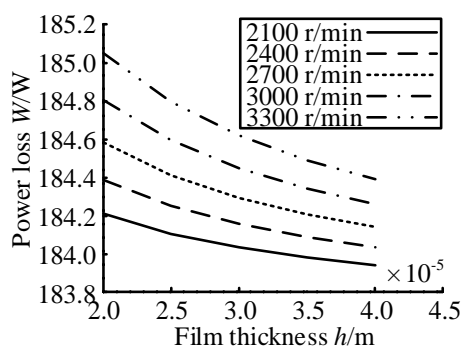

Fig. 11 Relationship between power loss and film thickness

\section{Conclusion}

(1) Bearing force is composed of hydrostatic bearing force and dynamic bearing force, and static performance is only affected by hydrostatic bearing force.

(2) Bearing capacity increases gradually with the increase of pump pressure and the film thickness, decreases with the increase of shadow pocket thickness, and has nothing to do with flow rate and rotational speed.

(3) Static stiffness increases gradually with increasing of pump pressure, decreases with increasing of shadow pocket thickness and film thickness, and has nothing to do with flow rate and rotational speed.

(4) Power loss increases gradually with increasing of flow rate, pump pressure and rotational speed, decreases with increasing of shadow pocket thickness and film thickness.

\section{Acknowledgement}

The project was financially supposed by National Natural Science Foundation of China (Grant No. 51075348), Hebei Provincial Natural Science Foundation of China (Grant No. E2011203151) and Research Fund for Doctoral Program of Higher Education of China (Grant No. 20101333110002).

\section{References}

[1] BRITO F P, MIRANDA A S, CLARO J C P, et al. The Role of Lubricant Feeding Condition on the Performance Improvement and Friction Reduction of Journal Bearings [J]. Tribology International. 2014, 72(0301679X): 65-82.

[2] WOJCIECH L, ARTUR O. Water-Lubricated Sintered Bronze Journal Bearings-Theoretical and Experimental Research [J]. Tribology Transactions. 2013, 57(1): 114-122.

[3] LONG X Y, YANG S M, YUAN G T, et al. Analysis of Hydrostatic Guideway Error based on Multi-Head Pump [J]. Advanced Materials Research. 2013, (712-715):2059-2064.

[4] WANG Y, PANG Z C. Effect of Design Gap of Quantitative Oil-supply Hydrostatic Bearing on Static and Dynamic Characteristics [J]. Machinery, 1990, 17(6): 17-19.

[5] LIU C X. Design of Constant Flow Control on Liquid Hydrostatic Guide-way [J]. Machine Tool \& Hydraulics, 2008, 36(7): 125-128.

[6] WANG H, CHEN X D. Design and Analysis of Constant Flow Control on Hydrostatic Guide-way [J]. Equipment Manufactring Technology, 2011, (7): 66-68. 
[7] GUO Y Y. Design of Hydrostatic Slide of Vertical Lathe [J]. Mechanical Engineer,, 2011, (6): 118-119.

[8] LIU J S. Design of Closed-type Hydrostatic Slide of Wheel Head of NC Cylindrical Grinder [J]. Precise Manufacturing \& Automation,, 2011, (4): 36-39.

[9] SUN G S, FENG M. Design of Hydrostatic and Dynamic Hybrid Bearing [M]. Beijing: World Publishing Corporation, 1993. 